\title{
Le contrôle des anabolisants dans la viande
}

\section{The survey of anabolic agents in meat}

\section{Bruno LE BIZEC, Philippe MARCHAND, François ANDRÉ}

LDH/NR École Nationale Vétérinaire - BP 50707 - 44307 NANTES Cedex 3 Tél : 0240687766 - Fax : 0240687878

(Reçu le 10 décembre 1999 ; accepté le 7 janvier 2000)

\section{$\boldsymbol{R E} \boldsymbol{E} U \boldsymbol{M E}$}

Le contrôle de l'usage de substances interdites en élevage est organisé et réglementé au niveau européen. Il est basé le plus souvent sur la recherche des molécules prohibées ou encore de leurs métabolites chez l'animal, dans leurs excréta, voire dans tout tissu pouvant être prélevé. Néanmoins, dans certaines situations particulières, viandes foraines ou d'importation par exemple, le seul moyen de contrôle efficace est la mise en évidence directe de la substance interdite dans la viande elle-même.

C'est la raison pour laquelle, une méthode permettant la recherche simultanée dans la viande (ou le foie) de nombreux composés anabolisants interdits a été développée au sein du Laboratoire National de Référence (LDH/LNR) (Ministère de l'Agriculture et de la Pêche), conformément aux exigences de la directive 96/23 EC.

La procédure analytique consiste tout d'abord en la lyophilisation du tissu prélevé avant extraction liquide-liquide. Deux hydrolyses successives autorisent respectivement la déconjugaison des métabolites de phase II (sulfates, glucuronides) et la saponification des esters de stéroïdes. Après partage des analytes en deux sous familles, les androgènesprogestagènes et les phénolstéroïdes, deux étapes d'extraction en phase solide ( $\mathrm{SPE} \mathrm{NH}_{2}$ et $\mathrm{SiOH}$ ) permettent la purification des analytes.

Une réaction spécifique de dérivation est dédiée à chacune des quatre sous familles de promoteurs de croissance extraites.

L'identification de 23 anabolisants différents est assurée en associant chromatographie en phase gazeuse et spectrométrie de masse, basse et haute résolution. Les limites de détection pour l'ensemble des analytes sont comprises entre 5 et $100 \mathrm{ng} / \mathrm{kg}(\mathrm{ppt})$.

\section{MOTS-CLÉS}

Anabolisants, résidus, viande, spectrométrie de masse.

\section{SUMMARY}

The survey of the illegal use of banned drugs including anabolic agents in food producing animals is regulated in the European Union. The compounds are usually looked for in animal feed, in urine or feces or in any animal tissue which may be easily sampled such as hair for example. However, in some cases, for retail or imported meat, the survey has to be performed by direct assay in the meat itself.

A method has been developed in the National Reference Laboratory for hormones (French Ministry of Agriculture) in order to assay simultaneously various anabolic agents according to the requirements of EC 96/23 Directive for meat (or liver) control. The meat sample is firstly lyophilised and extracted with organic solvents; phase II metabolites are hydrolysed and esters are saponified. Free compounds are extracted again thanks to various liquid-liquid and solid phase extraction.

The method allow the final identification of 23 various anabolic agents by gas chromatography coupled to mass spectrometry, either in the low and the high resolution mode detection levels are in the 5 and $100 \mathrm{ng}^{\mathrm{kg}} \mathrm{kg}^{-1}$ range. 


\section{Introduction}

L'interdiction, depuis 1989, d'utiliser les anabolisants en élevage dans les pays de l'Union Européenne a impliqué que se développe un système de contrôle. Deux types de réglementation ont vu le jour ; l'une concerne les plans d'échantillonnage, les espèces animales et les divers types de produits alimentaires à contrôler ainsi que l'organisation des laboratoires assurant ce contrôle ; il s'agit actuellement de la directive 96/23 EC (1), l'autre réglementation concerne les méthodes. De longue date, la commission européenne a proposé des critères de qualité auxquels doivent répondre les méthodes d'analyse ; aujourd'hui, la directive 93/256 EC (2) prévaut encore dans ce domaine, bien qu'elle doive être remplacée en 2000. Les méthodes qui relèvent de cette réglementation sont aussi bien celles qui ont pour objet la vérification des niveaux tolérés de résidus des substances autorisées en élevage que celles qui ont pour objectif la détection des traces de substances interdites en élevage, dont les anabolisants font partie. En ce qui concerne cette dernière famille de molécules, les exigences sont plus strictes en terme d'identification et de niveau concentrationnel de détection, de telle sorte que seule la spectrométrie de masse satisfait à cette contrainte.

La surveillance doit s'effectuer dès l'élevage des animaux, via le contrôle de leur alimentation. En outre, la preuve de l'administration de substances illégales peut être démontrée dans les excréta des animaux; à ce titre, I'urine est le substrat d'analyse privilégié, bien que les fèces soient parfaitement utilisés pour des molécules particulières. L'urine présente de nombreux avantages, simplicité de prélèvement, de conservation et de transport, analyte plus facile à traiter lors des premières phases d'extraction. C'est dans cette matrice biologique qu'ont été par conséquent développées les principales méthodes d'analyse en Europe (3), même si pour certains composés, tels les gestagènes, les matières fécales ont été utilisées ou le plasma sanguin pour le dosage des hormones naturelles.

Deux développements récents ont vu le jour dans le cadre de la recherche des substances interdites en élevage. Le premier concerne les méthodes d'analyses des phanères considérées comme un témoin à plus long terme de ce que l'animal a reçu, l'autre concerne l'analyse de la viande (ou des abats). Le contrôle de cette matrice est intéressant à plusieurs titres. Tout d'abord, il permet dans le cadre national de connaître la salubrité des denrées destinées à la consommation, de l'abattoir à l'assiette, via les étals. Il autorise en outre la vérification d'absence de résidus d'anabolisants de produits carnés importés des Pays Tiers, bien qu'ils soient accompagnés de documents officiels certifiant l'absence de telles substances.
A ces remarques, il convient d'ajouter que la recherche des substances interdites en élevage se doit d'être une recherche la plus ouverte possible, c'est à dire que la méthode proposée doit être capable de mettre en évidence le plus grand nombre de composés au plus bas niveau concentrationnel possible. Heureusement, les molécules efficaces sur la croissance des animaux font partie de familles connues et la distribution, même illégale, ne se fait que sur un nombre limité de substances. C'est dans cet objectif qu'a été développée entre autres une méthode de recherche d'anabolisants stéroïdiens dans la viande ou le foie décrite ici. Elle a été utilisée avec succès notamment dans le cadre de la surveillance des viandes importées des USA en 1999 (7).

\section{Matériel et méthodes Réactifs :}

Les réactifs et solvants sont de qualité analytique. Ils proviennent de chez Merck (Darmstadt, Allemagne) ou SDS (Peypin, France). Le suc d'Helix pomatia a été fourni par Biosepra (Villeneuve la Garenne, France), alors que les colonnes d'extraction en phase solide (silice-aminopropyl) sont disponibles chez SDS (Harbor city, CA, USA) ou (styrène-divinylbenzène ENVI-ChromP) chez Supelco (St Quentin Fallavier, France). Les réactifs de dérivation (MSTFA, MTBSTFA, TBDMCS et TMIS) proviennent de chez Fluka (Buchs, Suisse), le dithiothréitol (DTT) de chez Aldrich (Milwaukee, WI, USA). Les stéroïdes de référence ont été fournis par Steraloids (Wilton, NY, USA), Research Plus (Bayonne, NJ, USA) et par le RIVM (Bilthoven, Pays Bas) (Tableau I).

\section{Échantillons :}

Des échantillons de muscle de bovins non traités ont été utilisés comme témoins (blancs) ; ils ont été supplémentés à $0,5 \mathrm{ng} / \mathrm{g}$ avec les divers stérö̈des recherchés. En outre, des échantillons commerciaux ont été prélevés par les services d'inspection à différents postes d'inspections frontaliers; tous les échantillons sont conservés congelés à $-18^{\circ} \mathrm{C}$ avant analyse.

\section{Extraction et purification :}

Des échantillons de muscle ou de foie (15 g) sont lyophilisés puis pulvérisés ; tous les composés et leurs métabolites sont alors extraits avec $12 \mathrm{~mL}$ de méthanol et $15 \mathrm{~mL}$ de tampon acétate $(0,2 \mathrm{M}, \mathrm{pH} 5,2)$. Après évaporation du méthanol, $80 \mu \mathrm{L}$ de suc d'Helix pomatia sont ajoutés à la phase aqueuse et l'ensemble est soumis à incubation $15 \mathrm{~h}$ à $52^{\circ} \mathrm{C}(4)$, puis déposés sur une colonne d'extraction en phase solide de type ENVI-ChromP préconditionnée. Les composés interférants sont élués par $3 \mathrm{~mL}$ d'hexane et les composés 
Annales de Toxicologie Analytique, vol. XII, $\mathrm{n}^{\circ} 1,2000$

Tableau I : Liste des composés recherchés et leurs standards internes respectifs (les temps de rétention sont donnés à titre indicatif et sont exprimés en minutes).

\begin{tabular}{|c|c|c|c|c|c|c|}
\hline \multicolumn{4}{|c|}{ COMPOSÉS } & \multicolumn{3}{|c|}{ STANDARDS INTERNES } \\
\hline Groupe & Nom & Ions & $\mathbf{t}_{\mathbf{r}}$ & Nom & Ions & $\mathbf{t}_{\mathbf{r}}$ \\
\hline Stilbènes & $\begin{array}{l}\text { Cis-DES } \\
\text { Hexestrol } \\
\text { Diènestrol } \\
\text { Trans-DES }\end{array}$ & $\begin{array}{c}496 / 191 \\
249 \\
494 / 479 \\
496 / 191\end{array}$ & $\begin{array}{l}17.59 \\
19.12 \\
19.23 \\
19.38\end{array}$ & $\begin{array}{l}{\left[{ }^{2} \mathrm{H}_{4}\right] \text { Hexestrol }} \\
{\left[{ }^{2} \mathrm{H}_{6}\right] \mathrm{DES}}\end{array}$ & $\begin{array}{l}251 \\
502 \\
\end{array}$ & $\begin{array}{r}19.08 \\
19.32 \\
\end{array}$ \\
\hline Ac Réso. & $\begin{array}{l}\text { Zéranol } \\
\text { Taléranol }\end{array}$ & $\begin{array}{l}538 / 433 \\
538 / 433 \\
\end{array}$ & $\begin{array}{l}18.91 \\
19.08 \\
\end{array}$ & $\begin{array}{l}{\left[{ }^{2} \mathrm{H}_{4}\right] \text { Zéranol }} \\
{\left[{ }^{2} \mathrm{H}_{4}\right] \text { Taléranol }}\end{array}$ & $\begin{array}{l}542 \\
542 \\
\end{array}$ & $\begin{array}{l}18.88 \\
19.05 \\
\end{array}$ \\
\hline Estrogènes & $\begin{array}{l}17 \alpha \text {-estradiol } \\
17 \beta \text {-estradiol }\end{array}$ & $\begin{array}{l}416 / 285 \\
416 / 285 \\
\end{array}$ & $\begin{array}{l}17.30 \\
17.89 \\
\end{array}$ & {$\left[{ }^{2} \mathrm{H}_{3}\right] 17 \beta$-estradiol } & 419 & 17.87 \\
\hline Androgènes & $\begin{array}{l}17 \alpha \text {-trenbolone } \\
17 \beta \text {-trenbolone } \\
17 \alpha \text {-nandrolone } \\
17 \alpha \text {-testostérone } \\
17 \beta \text {-nandrolone } \\
17 \beta \text {-testostérone } \\
\text { Noréthandrone } \\
\text { Bolastérone } \\
\text { Méthyltestostérone } \\
\text { Ethinyltestostérone } \\
\text { Noréthandrolone }\end{array}$ & $\begin{array}{l}449 / 380 \\
442 / 380 \\
418 / 194 \\
432 / 209 \\
418 / 194 \\
432 / 209 \\
432 / 287 \\
460 / 445 \\
446 / 301 \\
456 / 301 \\
446 / 287\end{array}$ & $\begin{array}{l}15.13 \\
15.26 \\
16.70 \\
17.01 \\
17.30 \\
17.63 \\
18.42 \\
18.64 \\
18.75 \\
18.97 \\
19.97 \\
\end{array}$ & $\begin{array}{l}{\left[{ }^{2} \mathrm{H}_{2}\right] 17 \beta \text {-trenbolone }} \\
{\left[{ }^{2} \mathrm{H}_{3}\right] 17 \beta \text {-nandrolone }} \\
{\left[{ }^{2} \mathrm{H}_{2}\right] 17 \beta \text {-testostérone }} \\
{\left[{ }^{2} \mathrm{H}_{3}\right] \text { Méthyltestostérone }}\end{array}$ & $\begin{array}{l}421 \\
434\end{array}$ & $\begin{array}{r}15.25 \\
\\
17.27 \\
17.61 \\
18.72\end{array}$ \\
\hline Gestagènes & $\begin{array}{l}\text { Progestérone } \\
\text { Mégestrol } \\
\text { Chlormadinone } \\
\text { Médroxyprogestérone } \\
\text { Mélengestrol }\end{array}$ & $\begin{array}{l}458 / 443 \\
558 / 453 \\
578 / 473 \\
560 / 330 \\
570 / 555\end{array}$ & $\begin{array}{l}20.35 \\
20.92 \\
21.03 \\
21.07 \\
21.17\end{array}$ & $\begin{array}{l}\text { Norprogestérone } \\
{\left[{ }^{2} \mathrm{H}_{3}\right] \text { Mégestrol }} \\
\\
{\left[{ }^{2} \mathrm{H}_{3}\right] \text { Médroxyprogestérone }} \\
{\left[{ }^{2} \mathrm{H}_{3}\right] \text { Mélengestrol }}\end{array}$ & $\begin{array}{l}444 \\
561 \\
563 \\
573\end{array}$ & $\begin{array}{l}20.06 \\
20.88 \\
\\
21.03 \\
21.14\end{array}$ \\
\hline
\end{tabular}

d'intérêt par $14 \mathrm{~mL}$ du mélange hexane-diéthyl éther $(70: 30 ; \mathrm{v} / \mathrm{v})$. Les esters sont alors hydrolysés $30 \mathrm{~min}$ à $50^{\circ} \mathrm{C}$ avec $500 \mu \mathrm{L}$ de méthylate de sodium à $1 \%$ dans le méthanol après mise à sec de l'éluant. L'ajout de $1 \mathrm{~mL}$ de soude $(1 \mathrm{M})$ et de $8 \mathrm{~mL}$ du même mélange hexane-diéthyl éther permet la séparation des composés phénoliques (phenolstéroïdes, stilbènes et acides résorcyliques) (phase aqueuse) et des composés $\Delta 4$ 3 one (androgènes et gestagènes) (phase organique) (Fig 1).

\section{Purification des composés phénoliques :}

Ces derniers sont extraits de la phase aqueuse (préalablement neutralisée par $150 \mu \mathrm{L}$ d'acide acétique glacial) par $8 \mathrm{~mL}$ de diéthyl éther ; après mise à sec et reprise par $500 \mu \mathrm{L}$ du mélange hexane-dichlorométhane $(60: 40 ; \mathrm{v} / \mathrm{v})$ une purification sur colonne de silice conditionnée à l'hexane est effectuée (5). Les composés interférants sont élués par $3 \mathrm{~mL}$ du mélange hexane-acétate d'éthyle $(85: 25 ; \mathrm{v} / \mathrm{v})$ puis successivement les stilbènes par $13 \mathrm{~mL}$ du mélange hexane-acétate d'éthyle $(75: 25 ; \mathrm{v} / \mathrm{v})$ et les autres composés (ac. résorcyliques et phénolstéroïdes) par $13 \mathrm{~mL}$ du mélange hexane-acétate d'éthyle $(60: 40 ; \mathrm{v} / \mathrm{v})$. Ces deux frac- tions sont mises à sec et reprises dans $2 \mathrm{~mL}$ d'acétate d'éthyle-méthanol (80:20; v/v).

Chaque fraction subit une purification complémentaire par simple passage sur une colonne de type aminopropyle.

\section{Purification des composés $\Delta 4-3 o n e$ :}

Après évaporation de la phase organique hexane-diéthyl éther, le résidu sec repris par $500 \mu \mathrm{L}$ d'hexanedichlorométhane est appliqué sur une colonne de silice conditionnée à l'hexane. Après lavage avec $8 \mathrm{~mL}$ de mélange hexane-acétate d'éthyle $(75: 25 ; \mathrm{v} / \mathrm{v})$, les stéroïdes anabolisants sont élués par $13 \mathrm{~mL}$ d'hexane-acétate d'éthyle $(60: 40 ; v / v)$. Cette fraction est divisée en deux parties pour permettre des dérivations spécifiques de la trenbolone d'une part et des androgènes et progestagènes d'autre part.

\section{Dérivation :}

Après évaporation, la fraction androgènes-progestagènes est dérivée $40 \mathrm{~min}$ à $60^{\circ} \mathrm{C}$ par $15 \mu \mathrm{L}$ de mélange MSTFA-TMIS-DTT $(1000: 5: 5 ; \mathrm{v} / \mathrm{v} / \mathrm{w})$, alors que la fraction contenant la trenbolone et ses métabolites est dérivée par $10 \mu \mathrm{L}$ de mélange MSTFA- $\mathrm{I}_{2}(1000: 4$; v/v), 


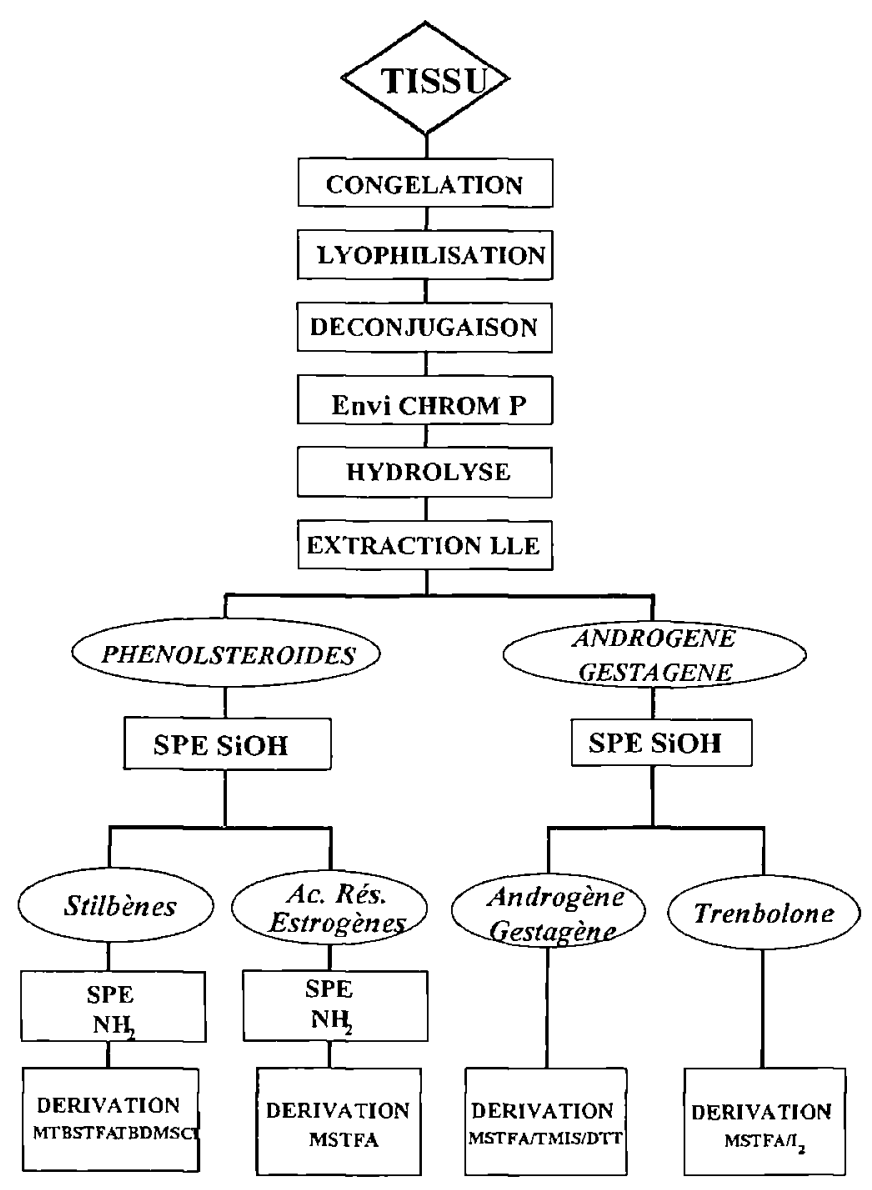

Figure 1 : Procédure analytique générale (SPE : Extraction en phase solide, LLE : Extraction liquide liquide).

la réaction est immédiate à température ambiante.

Les acides résorcyliques et phénolstérö̈des sont dérivés par $15 \mu \mathrm{L}$ de MSTFA à $60^{\circ} \mathrm{C}$ pendant 40 min et les stilbènes par $15 \mu \mathrm{L}$ de MTBSTFA-TBDMCS (100:1 ; $v / v)$ à $60^{\circ} \mathrm{C}$ pendant $75 \mathrm{~min}$.

\section{Analyse par chromatographie gazeuse couplée à la spectrométrie de masse :}

Les séparations chromatographiques sont effectuées classiquement sur colonne capillaire de type OV-1 (Ohio-Valley) à l'aide d'un chromatographe HP 6890, avec une programmation de $120^{\circ} \mathrm{C}(2 \mathrm{~min})$ à $250^{\circ} \mathrm{C}$ $\left(15^{\circ} \mathrm{C} \mathrm{min}^{-1}\right)$ puis $5^{\circ} \mathrm{C} \mathrm{min}^{-1}$ jusqu'à $300^{\circ} \mathrm{C}(8 \mathrm{~min})$.

L'analyse par spectrométrie de masse est faite en mode impact électronique (70 eV) acquisition SIM à l'aide d'un détecteur de masse HP 5973.

\section{Résultats et discussion}

\section{Extraction et purification :}

- En ce qui concerne la préparation des échantillons de viande, la lyophilisation permet l'obtention d'une poudre fine et homogène assurant une très bonne extraction des composés.
- La présence de formes conjuguées dans le tissu musculaire a été démontrée par l'analyse comparative avec ou sans hydrolyse d'échantillons de viande. Ces essais montrent une augmentation importante du taux d'estradiol $17 \alpha(50 \%$ env) et une légère augmentation de celui d'estradiol $17 \beta$ lorsqu'une hydrolyse est pratiquée. Cela prouve la présence de formes conjuguées dans le tissu musculaire et l'intérêt de l'étape d'hydrolyse pour la recherche de traces d'anabolisants stéroïdiens d'usage interdit dans la viande.

- L'extraction sur une phase mixte styrène et divinyl benzène en lieu et place des extractions classiques en phase solide de type $\mathrm{C}_{18}$ donne de meilleurs résultats lors des séparations chromatographiques en assurant une retenue plus forte des composés interférants.

- La forme particulière de certains analytes rend difficile leur analyse en CG-SM, ceci en raison d'une dégradation observable dans l'injecteur ou dans la colonne de chromatographie : c'est le cas des esters de progestagènes comme les acétates de mélengestrol ou de médroxyprogestérone. C'est pourquoi a été introduite une étape d'hydrolyse des esters à l'aide du méthylate de sodium à $1 \%$ dans le méthanol. Ce procédé libère les fonctions alcool des composés et améliore la réponse pour les progestagènes.

- Dans le but d'améliorer les chromatogrammes d'ions pour les stéroïdes endogènes, une méthode de séparation de ces groupes à été développée, sous la forme d'une simple partition liquide-liquide. Tous les composés à fonction phénol (estrogènes, stilbènes..) sont convertis en phénolates en milieu alcalin de telle sorte qu'ils restent en phase aqueuse en présence d'un solvant non polaire. Par ailleurs, tous les stéroïdes non phénoliques restent dans la phase organique. Ceci autorise une purification ultérieure par groupes et conduit à l'obtention de chromatogrammes plus limpides en terme d'interprétation.

- Les composés phénoliques sont purifiés à l'aide d'une chromatographie sur colonne de silice grâce à un gradient d'acétate d'éthyle dans l'hexane. A faible concentration d'acétate d'éthyle (15\%), les stilbènes peu polaires sont élués, suivis des estrogènes et acides résorcyliques à plus forte concentration d'acétate d'éthyle $(20 \%)$. Chacun de ces groupes est soumis à une purification complémentaire sur colonne de silice greffée aminopropyle, étape qui n'affecte en rien le rendement global d'extraction.

- Les androgènes et progestagènes ( $\Delta 4$-3one) sont purifiés d'une manière similaire.

\section{Dérivation :}

Une dérivation spécifique a été développée et appliquée pour chaque groupe dans un souci d'optimisation de la réponse (Tableau II). 
La dérivation classique des composés stéroïdiens avec le réactif MSTFA-TMIS-DTT a été appliquée aux androgènes et gestagènes. Les fonctions cétone et alcool sont respectivement converties en énol-TMS et éther-TMS. Le DTT évite la dégradation du réactif de dérivation.

La trenbolone et ses métabolites ne donnent pas de réponse répétable et sensible dérivés de cette manière, en raison de la présence de trois doubles liaisons conjuguées ; une dégradation du dérivé intervient au niveau du liner et de la colonne. C'est pourquoi une dérivation spécifique a été développée pour ce composé anabolisant, basée sur l'action du MSTFA en association avec l'iode $\left(\mathrm{I}_{2}\right)(6)$; le dérivé formé comprend la TMS énolisation du groupe 3-céto, la silylation du groupe 17-hydroxy et l'addition d'un groupement $\mathrm{N}\left(\mathrm{CH}_{3}\right) \mathrm{COCF}_{3}$ au noyau stérö̈dien en position 4 (Tableau II). Ce dérivé possède de bonnes propriétés chromatographiques et donne un spectre de fragmentation intéressant en mode impact électronique. En outre cette dérivation permet la différenciation des isomères $17 \beta$ et $17 \alpha$ de la trenbolone sur la base de leur spectre de masse, ce qui constitue une originalité en EI (Fig 2).
Tableau II : Composés dérivés selon le type de réaction de dérivation.
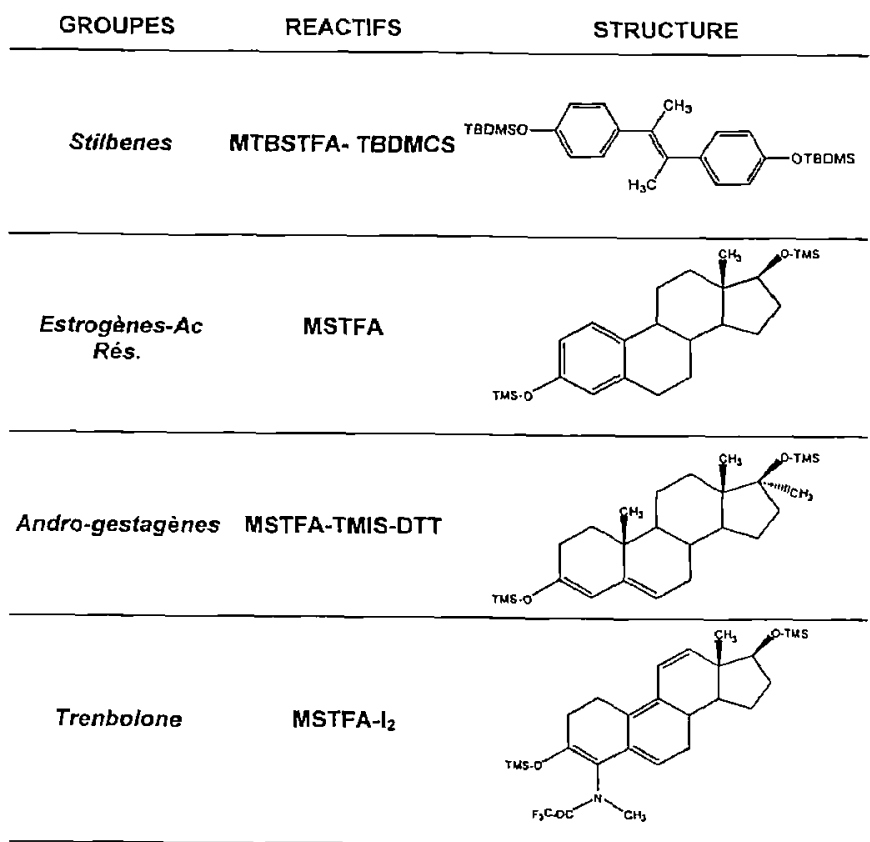

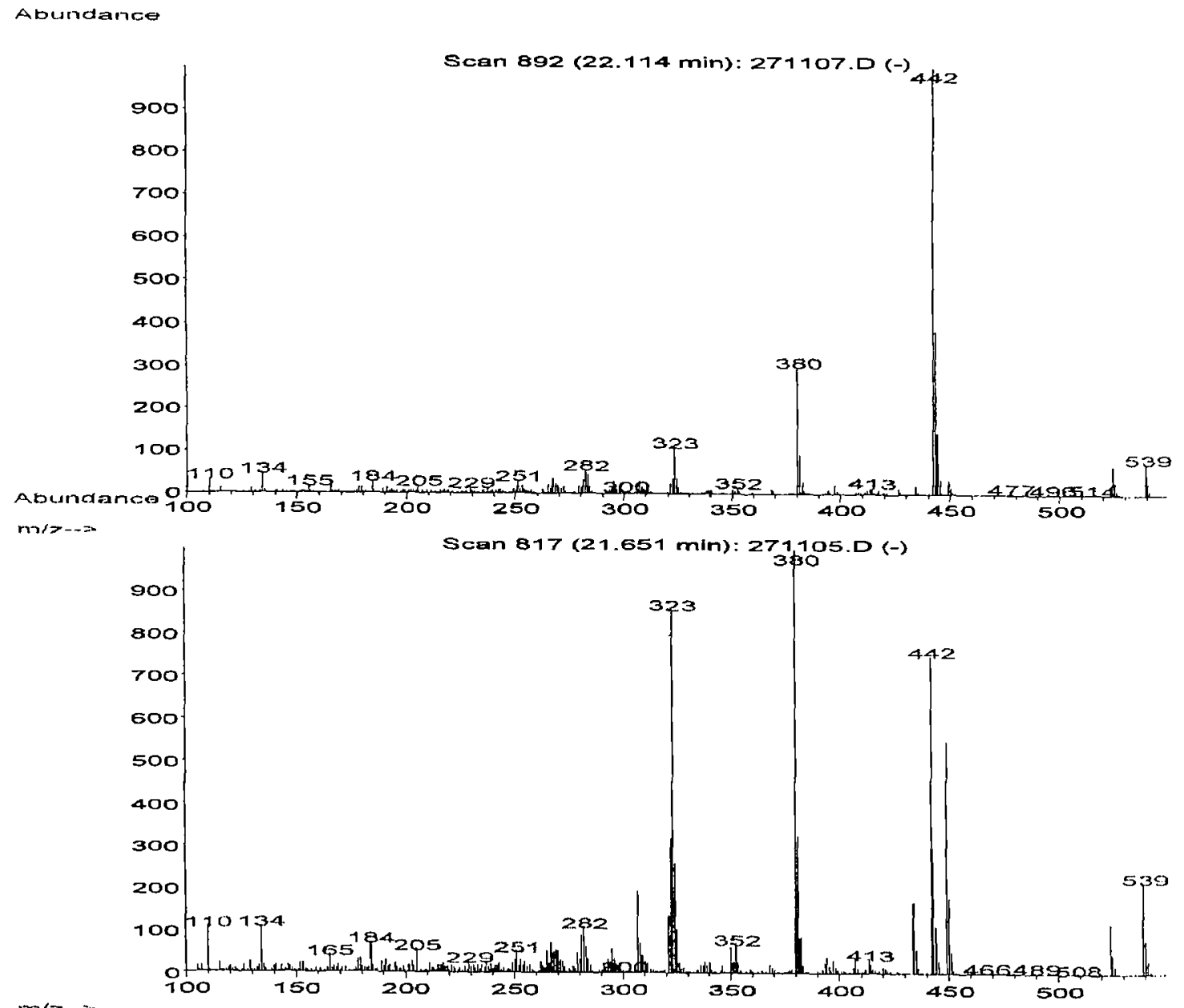

Figure $2:$ Spectre de masse de la $17 \alpha$-(haut) et $17 \beta$-trenbolone (bas) (Dérivation par $M S T F A / I_{2}$, ionisation par impact électronique, spectre de masse complet). 
La dérivation des acides résorcyliques et des composés à fonction phénol en 3 se réalise de façon modérée par Ie MSTFA ; il pourrait en être de même pour les stilbènes mais, compte tenu de leur relative volatilité, cela leur confère un temps de rétention chromatographique court qui les fait éluer rapidement avec certains contaminants ; c'est la raison pour laquelle l'obtention de dérivés di-TBDMS de plus haute masse est préférable, bien que la fragmentation soit réduite et qu'un faible nombre d'ions diagnostiques soit obtenu.

\section{Analyse d'échantillons supplémentés :}

Des essais ont été réalisés sur des échantillons de viande supplémentés à des concentrations dépendant du type de composé. A titre d'exemple, un échantillon supplémenté à $200 \mathrm{ng} / \mathrm{kg}$ (ppt) de trans-diéthylstilbestrol donne pour l'ion $\mathrm{m} / \mathrm{z} 496$ un rapport signal sur bruit de 30 . A la même concentration on observe un rapport estimé à 200 pour les $17 \alpha$ et $17 \beta$ nandrolone.

\section{Analyse d'échantillons contaminés :}

Des échantillons de viande provenant d'animaux traités avec divers anabolisants ou prélevés par les services d'inspection ont été analysés. Ainsi, la viande d'un animal ayant reçu une injection unique intramusculaire de
$250 \mathrm{mg}$ d'acétate de chlormadinone un mois avant abattage présente un chromatogramme d'ions permettant l'identification du composé conformément aux critères de la directive 93/256 EC en matière de temps de rétention, de nombre et de rapport des ions diagnostiques. La concentration de résidu peut être estimée à $2,5 \mu \mathrm{g} / \mathrm{kg}$ (Fig 3).

De même a pu être démontrée la présence de mélengestrol par le suivi de l'ion m/z 570 dans un échantillon de viande importée à une concentration de l'ordre de $1,5 \mu \mathrm{g} / \mathrm{kg}$ (Fig 4). Des échantillons de foie contenant de la trenbolone sous les formes $17 \alpha$ (env $9,0 \mu \mathrm{g} / \mathrm{kg}$ ) et $17 ß$ (env 1,5 $\mu \mathrm{g} / \mathrm{kg}$ ) (Fig 5) ou du zéranol (env 1,2 $\mu \mathrm{g} / \mathrm{kg}$ ) et son métabolite taléranol (env $0,9 \mu \mathrm{g} / \mathrm{kg}$ ) ont également pu être confirmés (Fig 6).

\section{Analyse de confirmation :}

Les procédures d'analyse décrites ci-dessus ont été mises en œuvre en utilisant un spectromètre de masse basse résolution. Néanmoins, la très faible concentration des résidus d'anabolisants dans la viande implique que l'identification définitive des composés se fasse à l'aide d'un spectromètre de masse magnétique à géométrie inverse type Madsuda (6). Ceci permet le respect des critères d'identification réglementaires en ce qui concerne les rapports d'ions notamment.

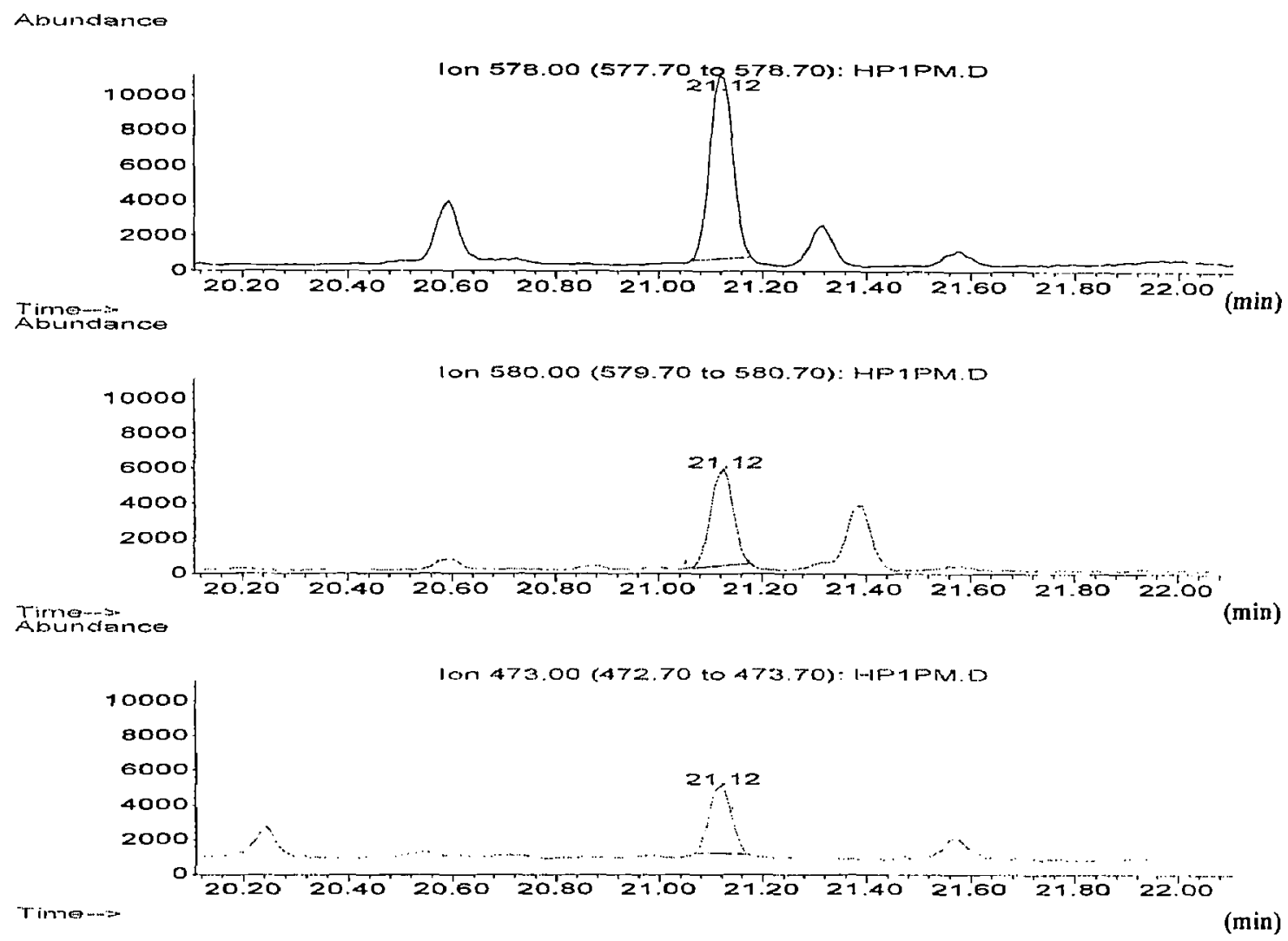

Figure 3 : Chromatogramme d'ions (SIM) d'un échantillon de viande positif en chlormadinone $(\mathrm{m} / \mathrm{z} 580,578,473, T R=21,12$ min). Dérivation MSTFA/TMIS/DTT, ionisation par impact électronique. Concentration estimée $: 2,5 \mu \mathrm{g} / \mathrm{kg}$. 
Annales de Toxicologie Analytique, vol. XII, $\mathrm{n}^{\circ} 1,2000$

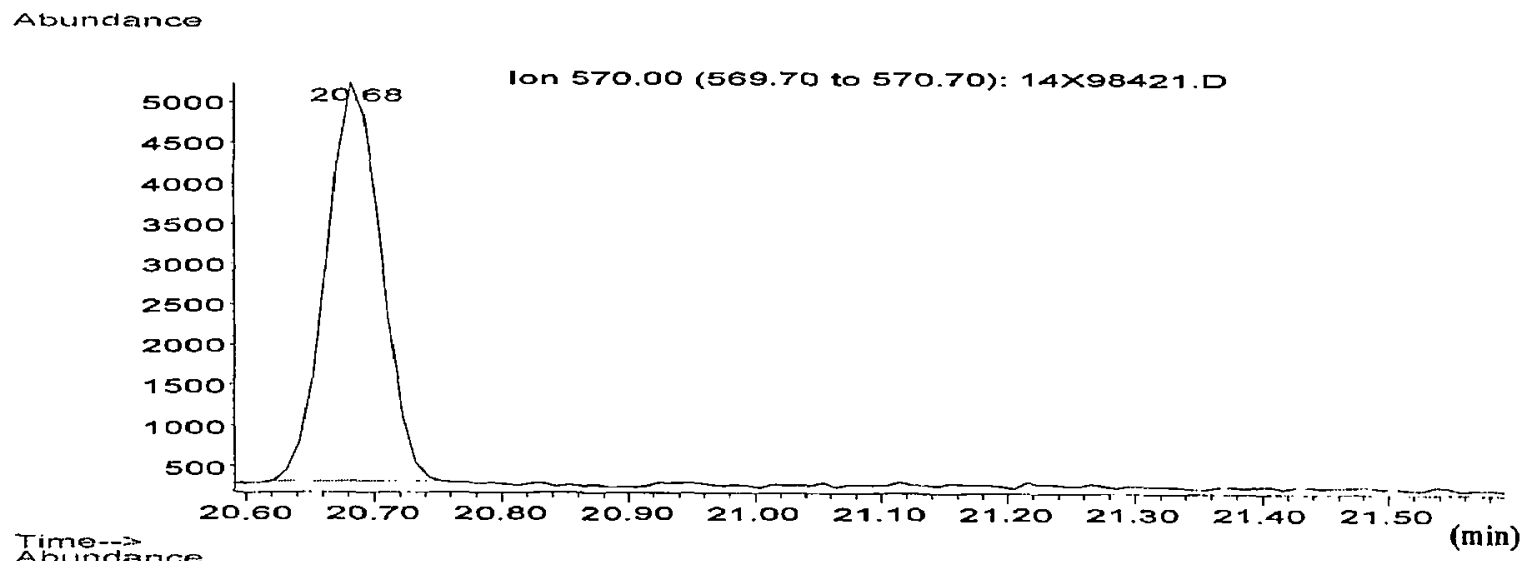

Time-indance

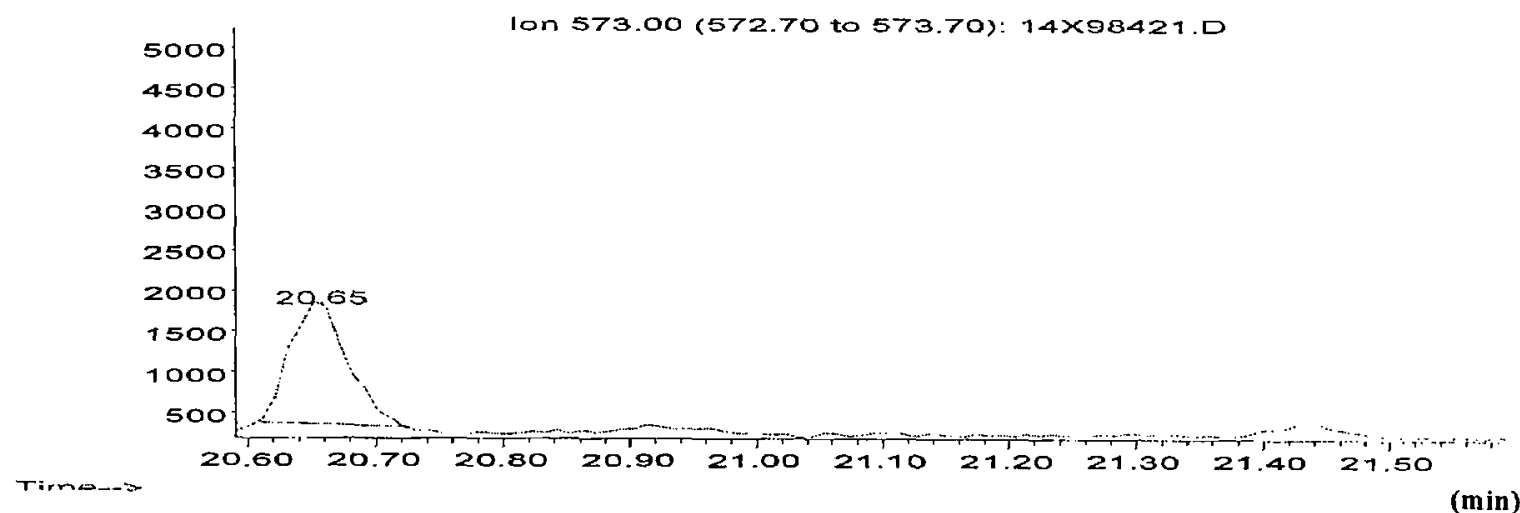

Figure 4: Chromatogramme d'ions (SIM) d'un échantillon de viande positif en acetate de mélengestrol (mélengestrol : m/z 570, $\left[{ }^{2} \mathrm{H}_{3}\right.$ Jmélengestrol $\mathrm{m} / \mathrm{z}$ 573, $\left.\mathrm{R}=20,65 \mathrm{~min}\right)$. Dérivation MSTFA/TMIS/DTT, ionisation par impact électronique. Concentration estimée : $1,5 \mu \mathrm{g} / \mathrm{kg}$.

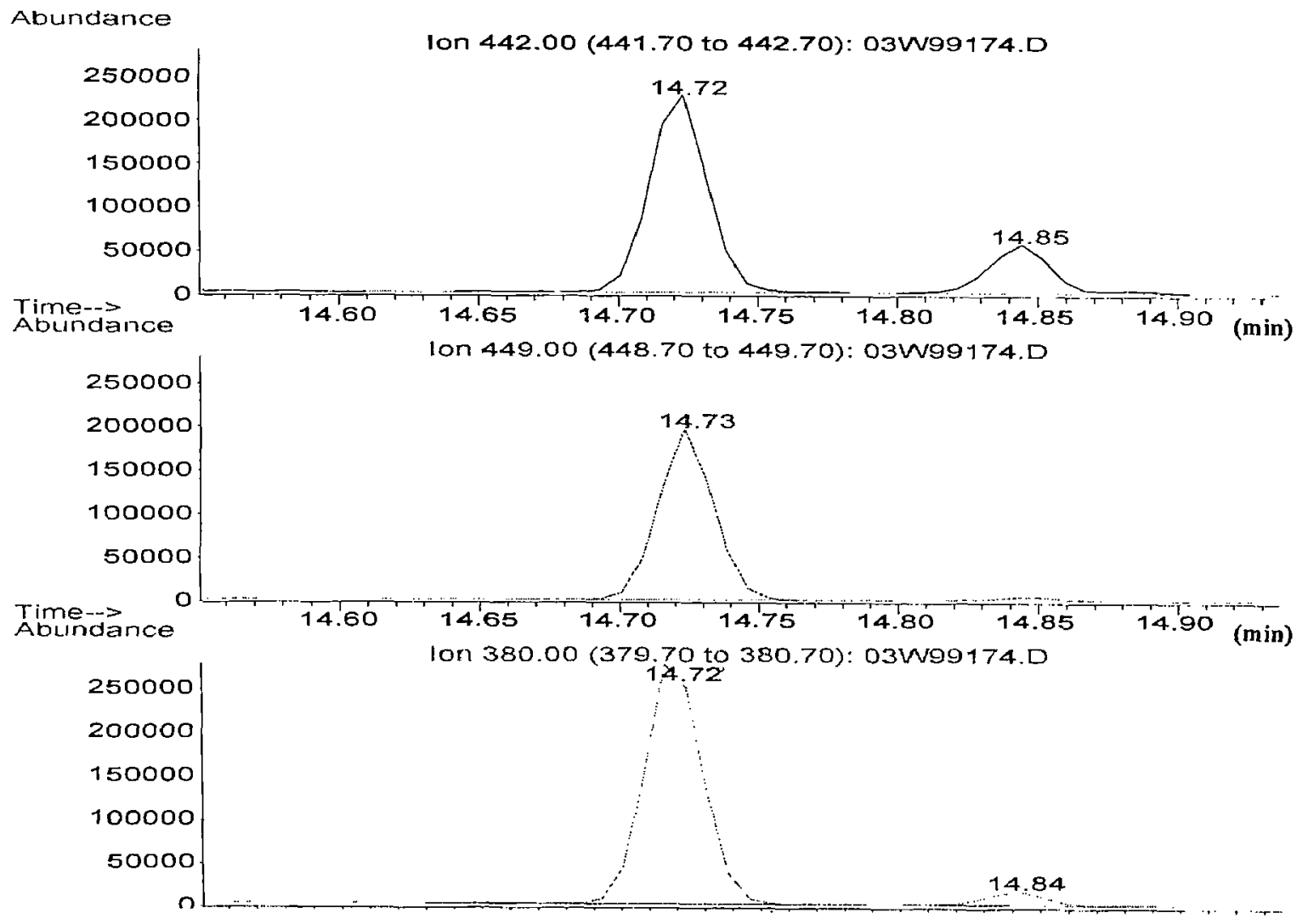

Figure 5 : Chromatogramme d'ions (SIM) d'un échantillon de foie positif en trenbolone (17 $\alpha$ - et $17 \beta$-trenbolone : $m / z 442$, 449, $380, R=14,73 \mathrm{~min}$ et $14,85 \mathrm{~min})$. Dérivation $M S T F A / I_{2}$, ionisation par impact électronique. Concentration estimée : $17 \alpha-$ trenbolone $=9,0 \mu \mathrm{g} / \mathrm{kg}$ et $17 \beta$-trenbolone $=1,5 \mu \mathrm{g} / \mathrm{kg}$. 


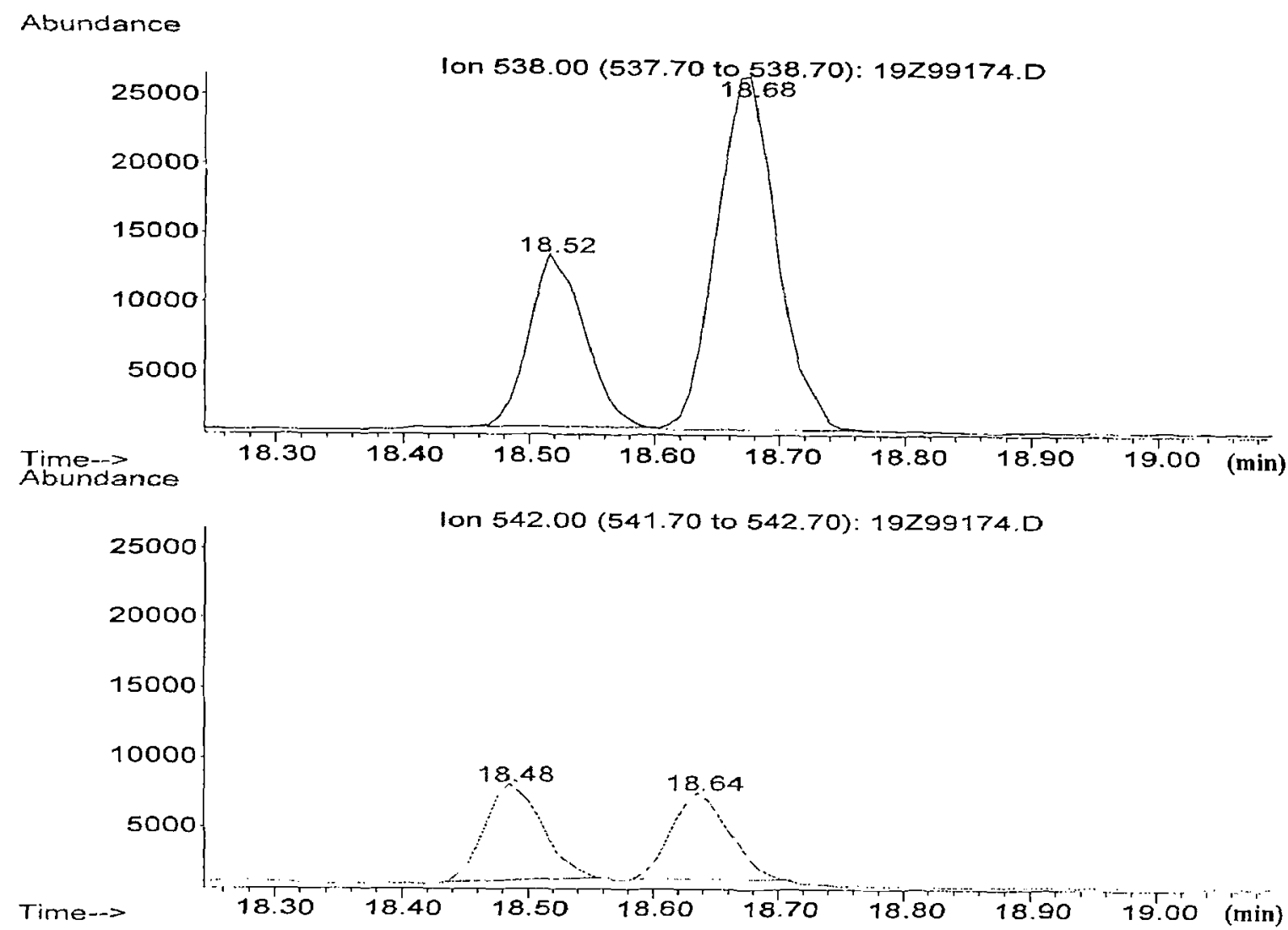

Figure 6 : Chromatogramme d'ions (SIM) d'un échantillon de foie positif en zéranoltaléranol (zéranol et taléranol : m/z 538 , $T R=18,52 \mathrm{~min}$ et $18 ; 68 \mathrm{~min} .\left[{ }^{2} \mathrm{H}_{4}\right]$ zéranol et $\left[{ }^{2} \mathrm{H}_{4}\right]$ taléranol : $\mathrm{m} / z$ 542, TR $=18,48 \mathrm{~min}$ et $\left.18,64 \mathrm{~min}\right)$. Dérivation $\mathrm{MSTFA}$, ionisation par impact électronique. Concentration estimée: zéranol $=1,2 \mu \mathrm{g} / \mathrm{kg}$ ét taléranol $=0,9 \mu \mathrm{g} / \mathrm{kg}$.

\section{Conclusion}

Le contrôle de la présence d'anabolisants dans la viande à partir d'une prise d'échantillon de l'ordre de la dizaine de grammes a été rendu possible pour un grand nombre de composés. La méthode développée comprend une extraction permettant de séparer les composés par groupes selon leurs propriétés physico-chimiques, stilbènes, acides résorcyliques et estrogènes d'une part, androgènes et progestagènes d'autre part. Cette approche permet des purifications complémentaires spécifiques et la mise en œuvre de dérivations optimisées pour chacune des sous familles.

La méthode a été validée sur des échantillons provenant d'animaux traités puis utilisée sur plusieurs centaines d'échantillons de viande prélevés officiellement, démontrant ainsi sa robustesse. Son efficacité a également été démontrée par la mise en évidence de plusieurs dizaines de cas positifs à l'acétate de mélengestrol, acétate de trenbolone ou encore zéranol, dans des échantillons provenant de pays tiers, ou encore d'acétate de chlorotestostérone et de chlormadinone dans des viandes d'animaux traités par nos soins.

\section{Remerciements :}

Ce travail a été réalisé dans le cadre d'un contrat de recherche de la Commission Européenne B67920/98/000826.

\section{Références}

1. Directive du Conseil 96/23/EC du 29 Avril 1996. JOCE (1996) N L $125,10$.

2. Décision du Conseil 93/256 du 14 avril 1993. JOCE (1993) $\mathrm{N}^{\circ} \mathrm{L} 118 / 64$

3. Heitzmann R.J. Residues in food producing animals and their products : reference materials and methods. Commission of the European Communities, Brussels, Report EUR 15127 EN Cy 1.2 (1993) $1-9$.

4. Le Bizec B., Montrade M.P., Monteau F., André F. Detection and identification of anabolic steroids in bovine urine by gas chromatography-mass spectrometry. Anal. Chim. Acta 275(1-2) (1993) 123-134.

5. Hartmann S., Steinhart H. Simultaneous determination of anabolic and catabolic steroid hormones in meat by gas chromatography mass spectrometry. J. Chromatogr. B 704 (1997) 105-117.

6. Maume D., Le Bizec B., Marchand P., Montrade M.P., André F. N-Methyl-N-alkylsilytrifluoroaceta$\mathrm{mie} / \mathrm{I}_{2}$ as new powerful derivatization reagent for anabolic steroid control. Analyst 123 (1998) 2645-2648.

7. Marchand P., Le Bizec B., Gasé C., Monteau F., André $F$. Vetra-trace detection of a wide range of anabolic steroids in meat by GC-MS. J. Chromatogr. A 867 (1999) 219-233. 